\title{
ON THE PECULIARITIES OF THE STAR LISTED AS NOVA \\ AURIGAE 1960-64 (KR AUR)
}

(Abstract)

MALINA POPOVA

Dept. of Astronomy, Bulgarian Academy of Sciences, Bulgaria

The variability of KR Aur was discovered in 1960 (Popova, 1960, 1961). As a scarce amount of plates was available it could not then be classified. Four years later on the ground of some new observations Hoffmeister supposed that KR Aur is a Nova of the RT Ser type, denoting it as Nova Aurigae 1960-64 (Hoffmeister, 1965, 1970).

We had the opportunity to make use of plate collections in the Sonneberg and Tautenburg Observatories (GDR) and in the Astronomical Institute of Sternberg, Moscow, for a photometric study of this star on about 400 plates taken from 1899 up to this year. It was shown that KR Aur is a blue variable with very unusual brightness variations and most probably is of a new type (Popova, 1965a, b, 1974).

In 75 years 7 rises of the brightness were established.

In Figure 1 observational data for the last 4 cycles are plotted. A length of about 6.5 years for these cycles may be derived. The star is more in maximum than in minimum brightness and according to the light curve it can be called more 'antinova' than 'nova'. Deep relative short minima attaining $18^{m}$ or fainter are followed by flat maxima with a mean magnitude of about 13.4 with fluctuations of 0.8 .

Noteworthy is the presence of considerable rapid rises and declines of the brightness. For instance in a day (2439442.5-443.5) the brightness declined by 1.8 (13.66-15.50). An outburst of $2^{m} \cdot 4$ at maximum light was also observed in 1963 . Five days after the brightness of 13.7 on three plates in a night the star had $11^{m} .3$,

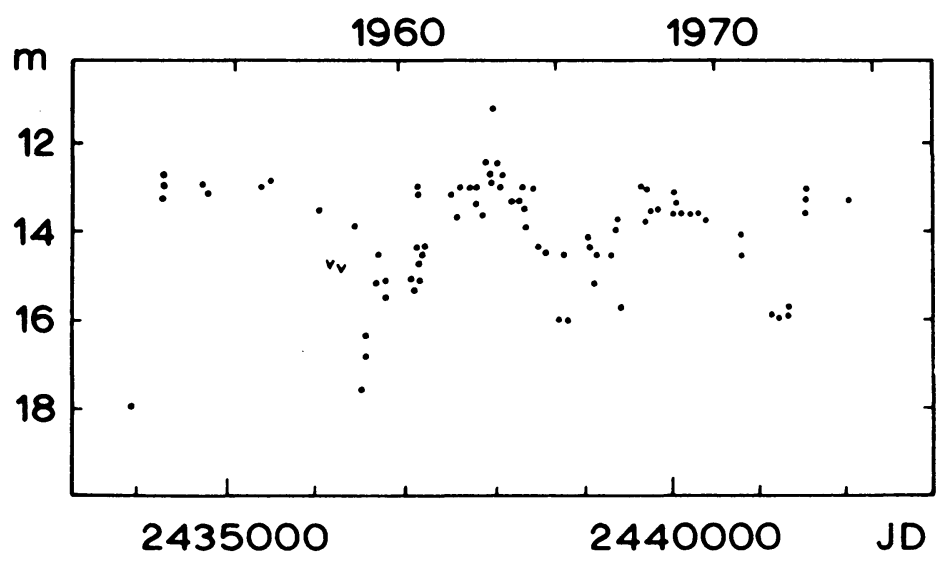

Fig. 1 . 
and 12 days later during the next observation it was $13^{m} \cdot 7$ too. On panchromatic plates the variable was of $1^{m} \cdot 2$ fainter at the outburst and also at normal maximum. Thus the brightness of KR Aurigae varies between 11.3 and $18^{m}$ or fainter.

It was worth-while to observe the spectrum of Nova Aur. An attempt was made by Preston, as it is reported in (Agenda and Draft Reports IAU, 1967). We took a low dispersion objective-prisma spectrum at maximum light with the $1-\mathrm{m}$ telescope $(1: 2: 1)$ of the Biurakan Observatory (Arm. SSR) (Popova, 1970). It was established that the star has a considerable UV excess. The absorption hydrogen lines are quite weak and hardly to be seen, but there is an emission of He II 4686. These preliminary results confirm the peculiarity of Nova Aurigae (1960-64).

I would like to emphasize that Nova Aur 1960-64 is now in maximum light and further observations, photometric and spectroscopic ones, with higher dispersion are very desirable.

\section{References}

Agenda and Draft Reports IAU: 1967, Prague, 532.

Hoffmeister, C.: 1965, Inf. Bull. IAU Var. Stars, No. 93.

Hoffmeister, C.: 1970, Veränderliche Sterne, Johann Ambrosius Barth, Leipzig.

Popova, M.: 1960, Mitt. über veränderliche Sterne 463.

Popova, M.: 1961, Astron. Nachr. 286, 81.

Popova, M.: 1965a, Inf. Bull. IAU Var. Stars, No. 97.

Popova, M.: 1965b, Var. Stars USSR 15, No. 5 (119) 534.

Popova, M.: 1970, Bull. Dept. Astron.' Bulg. Acad. Sci. 4, 51.

Popova, M.: 1974, Bull. Dept. Astron., Bulg. Acad. Sci. 7, in press. 